\title{
Astronomy with the Automated Patrol Telescope
}

\author{
B. D. Carter, M. C. B. Ashley, C. S. Bembrick, P. W. Brooks, \\ P. Mitchell and J. W. V. Storey
}

Department of Astrophysics \& Optics, School of Physics, University of New South Wales, PO Box 1, Kensington NSW 2033, Australia.

\begin{abstract}
The Automated Patrol Telescope (APT) is a wide-field CCD imaging telescope operated by the University of New South Wales at Siding Spring Observatory in Australia. The optical design employed resembles that of a Schmidt, but uses a 3-element lens to achieve a wide, corrected field of view. The APT was developed by extensively modifying the optical, mechanical and electronic systems of a Baker-Nunn satellite tracking camera. Telescope motion and operation of the CCD have been placed under computer control, allowing automated observations for longterm survey and monitoring projects. The APT has $0.5 \mathrm{~m}$ aperture $\mathrm{f} / 1$ optics which produce a $5^{\circ}$ flat field, of which a $2^{\circ} \times 3^{\circ}$ field is covered by the CCD currently installed. The telescope is being used for studies of stellar activity in open clusters and regions of star formation, and comet and minor planet investigations. A number of other projects for the APT are being considered, including searches for novae, supernovae in clusters of galaxies, and brown dwarfs.
\end{abstract}

\section{Introduction}

The Automated Patrol Telescope (APT) is a $0.5 \mathrm{~m}$ aperture instrument used for wide-field CCD imaging. The APT was developed by modifying a Baker-Nunn satellite tracking camera, donated to the University of New South Wales in 1982 by the Smithsonian Institution, U.S.A. The basic optical design of a Baker-Nunn camera is similar to a Schmidt, but uses a three-element corrector to produce an extremely wide field of view. In its original form, the optics could produce sharp images across a curved piece of film covering $5^{\circ} \times 12^{\circ}$.

The motivation behind the APT is to automate astronomical survey and monitoring observations. The aim is to use computer control of the telescope, its imaging system, and automated analysis of the incoming images to provide an extremely effective way of observing a wide area of sky, and over long timescales. As of February 1994, the APT has become operational after re-design and modification of its optics. The telescope is located at Siding Spring Observatory in Australia, and is housed in a roll-off roof building, allowing rapid all-sky access. Imaging with the telescope has been automated, and preliminary reduction of the raw images can be done as soon as they are taken. Work is in progress on automating both telescope slewing, and the detailed analysis of incoming images for variability studies. 


\section{Telescope specifications}

During its conversion to become the APT, the Baker-Nunn camera underwent extensive modifications to its mount and drive systems, and replacement of the film holder by a CCD camera. In recent years, the optics have been redesigned and altered to produce a wide and flat field, for imaging with large format CCDs, or mosaics. This work has been described by Carter et al. (1992) and Carter et al. (1994). The telescope specifications are summarised below.

Optics: The telescope has $\mathrm{f} / 1$ optics comprising a $0.5 \mathrm{~m}$ diameter, 3-element glass corrector lens, a $0.78 \mathrm{~m}$ diameter aluminised spherical primary mirror, and a plano-convex quartz field-flattening lens near the CCD. Five of the corrector lens surfaces are aspheric. Good throughput and star image quality are maintained from $350 \mathrm{~nm}$ to $1000 \mathrm{~nm}$. The optics produce a flat field $5^{\circ}$ in diameter.

Mounting and drives: The mount is equatorial with direct current servo motors, and absolute and incremental encoders on both axes. A servo system based around an Apple IIe microcomputer controls telescope slewing and tracking. The microcomputer and its telescope motion commands can be slaved to another computer.

Imaging system: A Wright Instruments CCD camera is used for direct imaging either unfiltered or through $\mathrm{B}, \mathrm{V}, \mathrm{R}$ or I broad-band filters. The camera is operated via a plug-in card in a personal computer. The CCD chip used is a thick, front-illuminated GEC CCD with a format of $770 \times 1152$ pixels, which equates to $1^{\circ} .95 \times 2^{\circ} .92$ on the sky. Each pixel is $22.5 \mu \mathrm{m}$ or 9 arcsec square, and has a large well depth of $400,000 \mathrm{e}^{-}$, minimising pixel saturation by bright stars. The CCD readout noise is $\sim 5 \mathrm{e}^{-}$rms.

Computer system: All aspects of the APT are to be controlled by a workstation computer. A DECstation 5000/120 workstation is used to operate and receive data from the CCD camera, via serial and ethernet links to the personal computer. Control of telescope motion from the workstation via the personal computer and Apple IIe is undergoing tests. The workstation can immediately display and reduce the raw images, and analysis of the reduced data can be done at the same time as the observations, using Figaro software. The 2 Mbyte frames are stored on a 5 Gbyte capacity exabyte tape.

\section{Photometric performance}

A calculation based on photon statistics predicts that the APT should detect $\mathrm{V} \approx 18$ stars in $100 \mathrm{~s}$ exposures, and detect $\mathrm{V} \approx 19$ magnitude stars in $300 \mathrm{~s}$, under typical dark-sky observing conditions. If the star is centred on a pixel, the detection is at the level of $10 \sigma$, but even when the star occupies two or four adjacent pixels, a positive detection still can be made. In addition, it is estimated that a signal-to-noise ratio of about 100:1 is possible, for photometry of 15 th magnitude stars recorded with a $100 \mathrm{~s}$ exposure. Sky noise dominates over readout noise in less than a second, and many exposures can be co-added to achieve the best signal-to-noise ratio. Preliminary observational tests have produced results consistent with the telescope's predicted performance. Stars can be detected to 18 th magnitude at the level of a few $\sigma$ in $100 \mathrm{~s}$, and a photometric precision of several percent appears possible to $\mathrm{V}=17$ in $300 \mathrm{~s}$. 


\section{Astronomical research programmes}

Astronomy with the APT will embrace a range of research projects using survey, monitoring and search observations. The APT offers CCD imaging with a large $2^{\circ} \times 3^{\circ}$ field, and the possibility of doing programmed sequences of photometry, or watching for transient events. For photometry, its modest $0.5 \mathrm{~m}$ aperture and large pixel size of 9 arcsec are offset by the very fast $f / 1$ optics, the high sensitivity of the $C C D$, the use of aperture photometry rather than profile fitting techniques, and the option to easily add many exposures when observing. At the time of writing, feasibility studies are being made for the following research projects:

\subsection{Stellar activity in open clusters and associations}

Stellar activity describes the analogue of solar activity that is observed in many low-mass stars. Studies of stellar activity are useful for understanding the structure and heating of stellar atmospheres, and can help us learn more about stellar rotation, evolution, and angular momentum loss. Young stars possess the most intense activity. The most energetic stellar flares observed occur on pre-main sequence stars, and flare activity is prevalent in many open clusters. Pre-main sequence stars show relatively large photometric variations indicative of star spots covering significant fractions of their surfaces, and chromospheric plages similar to those in solar active regions have been identified.

With its wide field of view, and facility for long-term photometric monitoring of cluster stars in B, V, R and I colours, the APT is well suited to photometric studies of activity in star clusters. In addition, its observations can complement spectroscopic investigations done with other telescopes. The APT will be used to observe stars in Galactic open clusters and regions of star formation, with research focussed on the following topics:

- Photometric and colour variability in pre-main sequence and early main sequence stars, and the role of starspots and accretion processes.

- Obtaining flares light curves, energies and statistics, to resolve present ambiguities, and provide data for studies of the relationship between flare activity and the evolution of low-mass stars.

- Surveying and explaining the systematic sequence in blue excesses observed among some very late-type, pre-main sequence stars.

- Investigation of the dichotomy in rotation rates that develops when stars evolve from the pre-main sequence to the main sequence.

\subsection{Solar system objects}

The APT will be part of the observing network being used to monitor the tails of comets, to provide information on the solar wind relevant to the Ulysses spacecraft mission. In addition, some cometary observations currently performed with the APT are for monitoring fluctuations in integrated coma magnitudes. A variety of minor planet observations are planned for the telescope. Photometry of known minor planets will be used to study asteroidal rotation. Confirmation 
of recently discovered objects will be made, when possible, and the chance appearance of unknown objects in APT frames will be investigated. A feasibility study will be conducted later in 1994, for an automated search for near-Earth asteroids, those objects which orbit so close to the Earth as to present a possible collision hazard.

\subsection{Clusters of galaxies}

The wide field of the APT makes it suitable for CCD imaging of clusters of galaxies. Some initial test frames have be obtained, for a survey of galaxyintegrated magnitudes and colours, in nearby clusters. These data will be used in conjunction with multicolour CCD imaging, at a much higher angular scale, of far more distant and less-evolved clusters. Comparison of the two datasets will provide a direct measurement of the observational effects of galaxy evolution.

\subsection{Other projects}

A number of other projects are under consideration for the APT. These projects include a dedicated search for supernovae in nearby galaxy clusters, searches for galactic novae, and a search for brown dwarfs. A supernova search in clusters would be very efficient, as the APT has a sufficient field to cover, in a single exposure, many of the galaxies in clusters such as those in Virgo or Fornax. Searches for galactic novae require effective coverage of a very wide part of the galactic plane, for perhaps a few events per year. A galactic nova search would be possible with the APT, but the gains must be considered against the observing time needed for other research projects. The possibility of a brown dwarf search has arisen through the work of Baillon et al. (1993). These authors promoted the feasibility of detections by observing gravitational lensing effects, even when angular resolution is relatively poor, if a sufficiently long-term observing programme is used.

\section{Conclusions}

The Automated Patrol Telescope is now operational, and work continues on increasing the level of automation of the telescope's operation. Preliminary observations and analysis for several different research projects have been carried out. The telescope will be used for stellar, solar-system and extragalactic observations, with initial observations being primarily for stellar activity, comet and minor planet studies. In future, technically more demanding automated patrols will be made, perhaps including a search for brown dwarfs.

\section{References}

Baillon P., Bouquet A., Giraud-Héraud Y. \& Kaplan J., 1993, A\&A, 277, 1

Carter B. D., Ashley M. C. B., Sun Y-S. \& Storey J. W. V., 1992, Proc ASA, 10,74

Carter B. D., Bembrick C. S., Ashley M. C. B. \& Mitchell P., 1994, Exp. Astron., in press 